1 This is a preprint of the final version in press at Consciousness and Cognition. (C) 2019. This

2 manuscript version is made available under the CC-BY-NC-ND 4.0 license

3 http://creativecommons.org/licenses/by-nc-nd/4.0/

4

5 Threat-induced Impulsivity in Go/Nogo Tasks: Relationships to Task-relevance of Emotional

$6 \quad$ Stimuli and Virtual Proximity

10 a Department of Psychology and Counseling, University of Chichester, Chichester, United

11 Kingdom.

$12{ }^{\mathrm{b}}$ Behavioral Science Institute, Radboud University Nijmegen, Nijmegen, The Netherlands.

$13{ }^{\mathrm{c}}$ Department of Psychiatry, Brain Center Rudolf Magnus, Utrecht University Medical Center, 14 Utrecht, The Netherlands.

$15{ }^{\mathrm{d}}$ Departments of Developmental and Experimental Psychology, Utrecht University, Utrecht, The 16 Netherlands

17 * Corresponding author: Thomas E. Gladwin, Address: Department of Psychology and 18 Counselling, University of Chichester, College Lane, Chichester, PO19 6PE, United Kingdom. 19 Tel.: +447895625183. Email: thomas.gladwin@gmail.com. 


\section{Disclosure Statements}

21 Declarations of interest: none.

\section{Abstract}

23 Threatening stimuli are thought to induce impulsive responses, but Emotional Go/Nogo task

24 results are not in line with this. We extend previous research by comparing effects of task-

25 relevance of emotional stimuli and virtual proximity. Four studies were performed to test this in

26 healthy college students. When emotional stimuli were task-relevant, threat both increased

27 commission errors and decreased RT, but this was not found when emotional stimuli were task-

28 irrelevant. This was found in both between-subject and within-subject designs. These effects

29 were found using a task version with equal go and nogo rates, but not with $90 \%-10 \%$ go-nogo

30 rates. Proximity was found to increase threat-induced speeding, with task-relevant stimuli only,

31 although effects on accuracy were less clear. Threat stimuli can thus induce impulsive

32 responding, but effects depend on features of the task design. The results may be of use in

33 understanding theoretically unexpected results involving threat and impulsivity and designing

34 future studies.

\section{Keywords}

36 Emotional Go-Nogo; Task-relevance; Faces; Impulsivity; Proximity

37 


\section{Introduction}

39 Threat-related stimuli induce tendencies to respond impulsively, in the sense of executing responses when they should be withheld (Hartikainen, Siiskonen, \& Ogawa, 2012;

41 Nieuwenhuys, Savelsbergh, \& Oudejans, 2012; Schutter, Hofman, \& Van Honk, 2008; van Peer,

42 Gladwin, \& Nieuwenhuys, 2018; Verbruggen \& De Houwer, 2007). Impulsive responding has

43 the advantage of speed, which may be essential, e.g., in life or death situations involving

44 predators, at the cost of reducing the time to complete sophisticated but slow cognitive

45 processing (Cunningham, Zelazo, Packer, \& Van Bavel, 2007; Nieuwenhuys \& Oudejans, 2012).

46 This may lead to suboptimal choices: For instance, in a simulated shooting situation, increasing

47 the threat associated with the task induced faster shooting and a bias to shoot versus refrain from

48 shooting (Nieuwenhuys et al., 2012). It is therefore important to understand threat-induced

49 impulsivity and the ways we measure it. One measure of impulsive responding is the stop signal

50 reaction time, SSRT (Bari \& Robbins, 2013; Verbruggen \& Logan, 2008). This is the time

51 required to cancel the execution of a response, when a stop signal is presented after a stimulus

52 initiating a response. As expected, threat has been found to increase the SSRT (van Peer et al.,

53 2018; Verbruggen \& De Houwer, 2007), i.e., threat makes it more difficult to inhibited response

54 execution, although this is not always found (Pawliczek et al., 2013; Sagaspe, Schwartz, \&

55 Vuilleumier, 2011). Also in line with a shift towards impulsive versus reflective responding, at a

56 neurobiological level threat increases the excitability of the corticospinal tract (Coombes et al.,

57 2009; Schutter et al., 2008) and reduces activity in regions associated with cognitive control

58 (Bishop, 2008; Oei et al., 2012).

59 Of particular interest to the current study, Go-Nogo tasks are frequently used to measure 60 impulsivity. Participants must respond quickly to one stimulus, and to refrain from responding to 
61 another stimulus. Threatening or highly arousing task-irrelevant distractor stimuli increase

62 commission errors (De Houwer \& Tibboel, 2010; Hartikainen et al., 2012), indicating that threat

63 reduced the ability to inhibit responses. This could reflect a shift in cognitive resources away

64 from the task (De Houwer \& Tibboel, 2010; Hartikainen et al., 2012). No effect on Go-stimulus

65 reaction time (RT) was found that would indicate a lowered response threshold; in one study, a

66 reversed effect was found (Brown et al., 2015). This is surprising, as it contradicts the theory-

67 based expectation that threat-induced commission errors should be caused by the shift towards

68 speed versus accuracy discussed above, i.e., reducing the evidence required for response

69 execution (Krypotos, Beckers, Kindt, \& Wagenmakers, 2015). This is an issue either for the

70 theory or for this method of measuring impulsivity.

71 The aim of the current paper is to address this issue, by exploring potentially important task

72 factors in the Go-Nogo task. In Study 1, the effect of task-relevance of emotional distractors was

73 tested. Previous work has shown that emotional stimuli have stronger effects when they must be

74 processed to perform the task, in terms of behavioural effects (Lichtenstein-Vidne, Henik, \&

75 Safadi, 2012; Spruyt, De Houwer, \& Hermans, 2009; Spruyt, Tibboel, De Schryver, \& De

76 Houwer, 2018) and neural responses (Pessoa, McKenna, Gutierrez, \& Ungerleider, 2002). The

77 automatic processes involved in emotional distraction may thus require at least some attention or

78 goal-relevance to be evoked, even though the subsequent effects on performance would not be

79 voluntary (Bargh, 1994; Bargh \& Ferguson, 2000; De Houwer, Teige-Mocigemba, Spruyt, \&

80 Moors, 2009). To extend this work to the Go-Nogo task, two versions of an emotional Go-Nogo

81 task were used. In one version, the emotional stimulus was a task-irrelevant distractor: Go versus

82 Nogo responses were signaled by probe stimuli independent from the emotional content. In the

83 other version, the emotional stimulus was the task-relevant probe stimulus: participants had to 
84 perform Go versus Nogo responses based on the emotional content of the stimuli (Megías,

85 Gutiérrez-Cobo, Gómez-Leal, Cabello, \& Fernández-Berrocal, 2017). This allowed us to test

86 whether task-relevant emotional, in this case threatening, stimuli would be more able to induce

87 the theoretically expected threat-enhanced impulsivity: more commission errors and lower Go-

88 RTs.

In Study 2, a further novel manipulation was introduced, namely the virtual relative proximity of

90

91

92

93

94

95

96

97

the stimuli. Proximity plays a central role in defensive responses (Blanchard et al., 2001;

Blanchard, Blanchard, \& Griebel, 2005; Blanchard, Griebel, Pobbe, \& Blanchard, 2011; Bradley, 2009; Kozlowska, Walker, McLean, \& Carrive, 2015; Mobbs et al., 2007). The change in defensive responses as a threat, e.g., a predator, comes closer is termed the defensive cascade: as a threat draws physically nearer, responses shift from freeze to flight to fight (Blanchard et al., 2005). At long distances, movement is suppressed (Bracha, 2004; Fanselow, 1986; Gladwin, Hashemi, van Ast, \& Roelofs, 2016; Roelofs, 2017; Sagliano, Cappuccio, Trojano, \& Conson, 2014); as the threat comes closer, flight responses occurs; and at very close range, fight responses are activated. Associated neurocognitive changes occur with increasing proximity to threat (Mobbs et al., 2007). The defensive cascade would appear to be related to the concept of defensive space, the minimal distance people desire to maintain between themselves and other people and potential threats, i.e., before defensive responses are activated (Graziano \& Cooke, 2006; Hayduk, 1983). Exposure to aggression (Vagnoni, Lewis, Tajadura-Jiménez, \& Cardini, 2018), anxiety (de Vignemont \& Iannetti, 2015; Sambo \& Iannetti, 2013) and psychoticism (McGurk, Davis, \& Grehan, 1981) have been shown to be related to a larger defensive space. Further, using fMRI study, veterans with anger and aggression problems showed abnormal brain activation in the cuneus, a region associated with the processing of emotionally salient stimulus 
107 features, when stimuli appeared closer versus further away (Heesink et al., 2017). Thus, the 108 impulsivity expected to occur when confronted with threat could interact with perceived 109 proximity. In Study 2 therefore, images were scaled to be larger or smaller to generate the 110 impression of being closer or further away from the participant. This is termed "zoomed-in"

111 versus "zoomed-out" below, but we note that there was no zooming animation: images were only 112 relatively large or relatively small, within the task. Note that the relative rather than absolute size 113 of a stimulus is likely important for whether a stimulus is perceived as far away or close, as the 114 absolute size has little meaning for an on-screen emotional stimulus in this context. Task115 relevance was also manipulated as in Study 1. We expected that stimuli appearing closer to 116 participants would enhance threat-induced effects on impulsivity.

117 In Study 3, data are presented in which the hypotheses of Study 1 were tested again, but using a 118 within-subject design in which all participants performed both the task-relevant and task119 irrelevant tasks.

120 In Study 4, the same within-subject design as in Study 3 was used, but with increased 121 proportions of go versus no-go trials (90\% versus $10 \%)$. In the previous studies, go and no-go 122 trials were equally likely. We note some reasons to use the 50-50 distribution, in particular for 123 the aims of the current research questions on interactions with threat stimuli. First, testing 124 whether threat-stimuli indeed induce impulsive responses does not depend on having a prepotent 125 response induced by the non-emotional manipulation of go-likelihood. Second, the 50-50 126 distribution avoids the disadvantage of a relatively small number of trials in the no-go condition. 127 Third, in the task-relevant version of the task, unequal go- and nogo-frequencies would result in 128 strongly differing block-contexts, which would be confounded with trial type; and hence, results 129 would be difficult to interpret. That is: threat-go trials only occur in threat-go blocks, in which 
130 participants would be exposed to primarily threatening stimuli; while on threat-nogo blocks,

131 most stimuli would be non-threatening. Fourth, unequal go and nogo distributions have the

132 disadvantage of confounding the nogo-manipulation with frequency and hence processes such as

133 expectation or attention, which could also conceivably interact with emotional stimuli. Finally, it

134 is not necessarily methodologically optimal to have a higher baseline level of impulsivity

135 induced by go-frequency; this could for example lead to ceiling effects on commission errors and 136 reduce the ability to detect additional emotional effects. However, Go-Nogo studies have tended

137 to use increased proportions of go-trials to the aim of increasing response tendency, and the final 138 Study may provide a possibly informative closer comparison to the existing literature.

139 Study 1

140 2. Method

141 2.1.Participants

142 Healthy participants were recruited and received study credits or a monetary reward for

143 completing the study. Participants gave informed consent. The study was approved by the ethics

144 review board. An analytical sample of 135 participants ( 88 female, 47 male, 23 years, $S D=7.1$ )

145 completed the experiment with performance indicating at least minimal task engagement,

146 quantified as accuracy over .5 in all analyzed trial types, excluding, for instance, participants

147 who simply executed go responses without paying attention $(n=2$ participants were removed

148 who did not reach the criterion).

\section{2.2. Emotional Go/Nogo Task (emoGNG)}

150 The tasks were programmed using HTML5, JavaScript and PHP. Randomization used the

151 seedrandom script by David Bau (https://github.com/davidbau/seedrandom). For each 
152 participant, the identifier assigned to them by the participant-pool system was converted to the

153 numerical random-seed for the module. Software is available on request by emailing the

154 communicating author. We acknowledge that a general limitation of online studies is some loss

155 of control relative to a laboratory setting; however, online studies have been shown to be a valid

156 method for psychological tasks (Chetverikov \& Upravitelev, 2016; van Ballegooijen, Riper,

157 Cuijpers, van Oppen, \& Smit, 2016).

158 Facial stimuli subtended around 7.5 degrees visual angle; the precise visual angles varied 159 depending on participants' screen size. Text stimuli had a visual angle of around 0.5 degrees. 14

160 pairs (neutral and angry) of computer-generated male faces were used from the Bochum

161 Emotional Stimulus Set (Thoma, Soria Bauser, \& Suchan, 2013).

162 The task consisted of 10 blocks of 48 trials (see Figure 1 for an illustration). Each participant 163 performed one of two versions, with either task-relevant or task-irrelevant emotional stimuli. In 164 both versions, trials began with a white fixation cross, for 250, 300, or $350 \mathrm{~ms}$. Subsequently, a 165 stimulus was presented consisting of an angry or neutral face stimulus and a small $x$ or $o$ symbol, 166 placed at a random location on the face. In the Task-Relevant version, participants were 167 instructed either to press space when an angry face appeared and to do nothing when a neutral 168 face appeared; or to press space when a neutral face appeared and to do nothing when an angry 169 face appeared. In the Task-Irrelevant version, participants were instructed either to press space 170 when an $x$ appeared and to do nothing when an $o$ appeared; or to press space when an $o$ appeared 171 and to do nothing when an $x$ appeared. In both conditions, the Go/Nogo mapping instructions 172 alternated per block. Participants had $600 \mathrm{~ms}$ to respond before the stimuli disappeared.

173 Feedback was presented after incorrect responses for $400 \mathrm{~ms}$ : A red "Incorrect!", or a red “Too 174 late!" 
$175<$ Figure 1>

177 Go and Nogo trials were equally frequency. Although previous Go-Nogo tasks have often used 178 lower probabilities for Nogo stimuli, to the aim of increasing response likelihood and hence the 179 probability of commission errors, please note that equal probabilities do not threaten evidence for 180 threat-induced impulsivity (and the results will indeed show that relatively infrequent Nogo trials 181 are not necessary to find such effects). A further advantage of equal probabilities is that there is 182 no confound between stimulus type and frequency.

\section{$183 \quad 2.3$. Procedure}

184 Inclusion proceeded via an online participant-pool system. Participants could sign up for the 185 study based on a brief description, after which they could read the extensive information and 186 decide whether to continue. Participants performed one of the emoGNG versions selected at 187 random. Other questionnaires and tasks were performed in the same session that were related to 188 other studies.

\subsection{Preprocessing and Statistical Analyses}

190 The first block of the task, the first four trials per block and trials following errors were removed 191 as these were considered to potentially deviate from normal task performance. Analyses were 192 performed in order to test effects per task as well as to compare the effects between tasks. Effects 193 per task were tested with a repeated measures ANOVA. The analyses were performed with the 194 dependent variables median RT, and the asin-square transformation of mean accuracy scores. 195 Median RTs were used to avoid effects of outliers which would require arbitrary cut-offs using 196 the mean. The transformation of the mean accuracy scores was used to normalize the 197 distribution. For RT, only go trials were included in the analysis. The within-subject factor was 198 Threat (Angry face versus Neutral face). For accuracy, the within-subject factors were Threat 
199 and Go (Go versus Nogo). In a subsequent mixed design ANOVA, task version was used as an 200 additional between-subject variable to test interactions involving task version. Note that we 201 chose to present the results for each task separately, to prevent the presentation of information 202 per task depend on the binary outcome of interactions involving the task version. All data and 203 statistical output are available on request.

\section{3. Results}

20566 participants performed the task-irrelevant emoGNG, and 69 participants performed the task206 relevant emoGNG. Descriptive statistics are presented in Table 1. 
208 Table 1. RT and accuracy on the emoGNG

209 1A. Reaction time on Go trials

\begin{tabular}{lll} 
Task version & Emotion & RT (SD) \\
\hline Task-irrelevant & Neutral & $449(29)$ \\
& Angry & $450(31)$ \\
Task-relevant & Neutral & $428(33)$ \\
& Angry & $419(30)$
\end{tabular}

211 1B. Accuracy

\begin{tabular}{llll} 
Task version & Emotion & Go/Nogo & Accuracy \\
\hline Task-irrelevant & Neutral & Nogo & .93 \\
& & Go & .94 \\
& Angry & Nogo & .92 \\
& & Go & .94 \\
Task-relevant & Neutral & Nogo & .91 \\
& & Go & .92 \\
& Angry & Nogo & .88 \\
& & Go & .93
\end{tabular}

212

213 Note. Mean and standard deviation of reaction time in ms and mean accuracy in proportion correct per

214 condition of the emoGNG over participants. Task version refers to task-relevance of the emotional

215 expression of the faces (Neutral or Angry).

216 


\section{3.1. Task-Irrelevant emoGNG}

218 There was no effect of Threat on RT $(p=.48)$ and no interaction between Go and Threat on

219 accuracy $(p=.092)$. Go trials were more accurate than Nogo trials, $F(1,65)=11, p=.0013, \eta_{\mathrm{p}}^{2}$

$220=0.15(.94$ versus .92$)$.

\section{3.2. Task-Relevant emoGNG}

222 On RT, there was an effect of Threat, $F(1,68)=15, p=.00027, \eta_{\mathrm{p}}{ }^{2}=0.18$, responding to Angry

223 faces being faster than responding to Neutral faces ( $419 \mathrm{~ms}$ versus $428 \mathrm{~ms}$ ).

224 On accuracy, there was an interaction between Go and Threat, $F(1,68)=21, p<.0001, \eta_{\mathrm{p}}{ }^{2}=$

225 0.24. This was due to lower accuracy for Angry than Neutral faces on Nogo trials, $F(1,68)=19$,

$226 p<.0001, \eta_{\mathrm{p}}{ }^{2}=0.22$ (.88 versus .91 proportion correct), and higher accuracy for Angry than

227 Neutral faces on Go trials, $F(1,68)=19, p=.044, \eta_{\mathrm{p}}{ }^{2}=0.058$ (.93 versus .92). Further, Go trials

228 were more accurate than Nogo trials, $F(1,68)=20, p<.0001, \eta_{\mathrm{p}}{ }^{2}=0.22$ (.92 versus .90$)$.

229

230

231

232

233

234

235

236

237

238

239

\subsection{Between-Task Comparisons}

The above difference in effects between the tasks were formally tested using a mixed design ANOVA. On RT, the interaction between Task version and Threat was significant, $F(1,133)=$ $13, p=.00052, \eta_{\mathrm{p}}^{2}=0.087$. No task-related interaction reached significant on accuracy, although the Task $\mathrm{x}$ Go $\mathrm{x}$ Threat interaction approached significance $(p=.056)$.

\section{Discussion}

The aims of Study 1 were to provide further information on whether threatening social stimuli induce impulsivity and determine what the effect is of using a task in which the emotional cues are task-relevant versus task-irrelevant. Effects involving threat were only found for the TaskRelevant version. Most importantly, a speeding effect was found on RTs on go trials. Using taskirrelevant emotional cues or distractors was also not previously found to affect RT on go-trials 
240 (De Houwer \& Tibboel, 2010; Hartikainen et al., 2012). Making the emotional stimuli task-

241 relevant appeared to allow them to induce impulsivity as detected via speeding, similarly to

242 effects of task-relevance (although we note that the precise meaning of "task-relevance" varies)

243 in other emotional tasks (Lichtenstein-Vidne et al., 2012; Spruyt et al., 2009, 2018).

\section{Study 2}

245 2. Method

\section{2.1. Participants}

247 Healthy participants were recruited and received study credits or a monetary reward for

248 completing the study, which was performed fully online. Participants gave informed consent and 249 the study was approved by the local ethics review board. 173 participants (151 female, 22 male; 250 mean age $20, S D=3.3$ ) completed the experiment with performance indicating at least minimal 251 task engagement, quantified as accuracy over .5 in all analyzed trial types $(n=2$ participants 252 were removed).

\section{2.2. Proximity version of the Emotional Go/Nogo Task (proxemoGNG)}

254 The proxemoGNG consisted of 9 blocks of 40 trials. Trials were identical to those of the

255 emoGNG, with the exception of a random "zoom-in" effect that occurred with 0.5 probability on

256 all trials. Note for clarity the zoom did not involve a movement animation: stimuli were simply

257 presented at different sizes. The facial visual stimuli subtended around 7.5 degrees visual angle, 258 except when zoomed-in in which case the angle was 15 degrees (as above, the precise visual 259 angles will have varied somewhat). The proxemoGNG was also presented in either a Task260 Relevant and Task-Irrelevant version. 


\section{2.3. Procedure}

262 Inclusion proceeded via an online participant-pool system. Participants could sign up for the

263 study based on a brief description, after which they could read the extensive information and

264 decide whether to continue. Participants performed the Task-Relevant or the Task-Irrelevant

265 version of the proxemoGNG, selected at random.

\section{2.4. Preprocessing and Statistical Analyses}

267 The first block of the task, the first four trials per block, and trials following errors were

268 removed. Analyses were performed in order to test effects per task as well as to compare the

269 effects between tasks. Effects per task were tested with a repeated measures ANOVA. The

270 analyses were performed with the dependent variables median RT and the asin-square

271 transformation of accuracy scores. For RT, only go trials were included in the analysis. The

272 within-subject factors were Proximity (Zoomed-In versus Zoomed-Out) and Threat (Angry face

273 versus Neutral face). For accuracy, the within-subject factors were Proximity, Threat and Go (Go

274 versus Nogo).

275 In a subsequent mixed design ANOVA, task version was used as a between-subject variable to 276 test interactions involving task version.

\section{3. Results}

27889 participants performed the task-irrelevant proxemoGNG, and 84 participants performed the 279 task-relevant proxemoGNG. Descriptive statistics are presented in Table 2. 
281 Table 2. RT and accuracy on the proxemoGNG

$282 \quad 2 \mathrm{~A}$. RT on Go trials

\begin{tabular}{llll} 
Task version & Emotion & Proximity & RT (SD) \\
\hline Task-irrelevant & Neutral & Far & $457(31)$ \\
& & Near & $453(32)$ \\
& Angry & Far & $457(32)$ \\
Task-relevant & Near & $452(31)$ \\
& Neutral & Far & $434(37)$ \\
& & Near & $433(36)$ \\
& Angry & Far & $436(37)$ \\
& & Near & $413(37)$
\end{tabular}

283

2842 2B. Accuracy

\begin{tabular}{|c|c|c|c|c|}
\hline Task version & Emotion & Go/Nogo & Proximity & Accuracy \\
\hline \multirow[t]{8}{*}{ Task-irrelevant } & Neutral & Nogo & Far & .94 \\
\hline & & & Near & .94 \\
\hline & & Go & Far & .94 \\
\hline & & & Near & .95 \\
\hline & Angry & Nogo & Far & .93 \\
\hline & & & Near & .93 \\
\hline & & Go & Far & .94 \\
\hline & & & Near & .95 \\
\hline \multirow[t]{8}{*}{ Task-relevant } & Neutral & Nogo & Far & .93 \\
\hline & & & Near & .91 \\
\hline & & Go & Far & .91 \\
\hline & & & Near & .92 \\
\hline & Angry & Nogo & Far & .86 \\
\hline & & & Near & .91 \\
\hline & & Go & Far & .92 \\
\hline & & & Near & .94 \\
\hline
\end{tabular}

285

286 Note. Mean and standard deviation of reaction time in ms and mean accuracy in proportion correct per 287 condition of the proxemoGNG over participants. Task version refers to task-relevance of the emotional 288 expression of the faces (Neutral or Angry). Proximity refers to whether the face presented on the trial 289 was zoomed in (Near) or not (Far). 


\subsection{Task-Irrelevant proxemoGNG}

On RT, the only significant effect was of Proximity, $F(1,88)=9.9, p=.0022, \eta_{\mathrm{p}}^{2}=0.10$, zoomed-in stimuli evoking a faster response than zoomed-out stimuli (453 ms versus $457 \mathrm{~ms}$ ).

On accuracy, the only effect was of Go, $F(1,88)=7.7, p=0.0069, \eta_{\mathrm{p}}^{2}=0.080$, Go-responses being more accurate than Nogo-responses (.95 versus .94$)$.

\subsection{Task-Relevant proxemoGNG}

On RT, effects were found of Threat, $F(1,83)=30, p<.0001, \eta_{\mathrm{p}}{ }^{2}=0.26$, Angry faces evoking faster responses than Neutral faces (424 ms versus $433 \mathrm{~ms}$ ); Proximity, $F(1,83)=54, p<.0001$, $\eta_{\mathrm{p}}{ }^{2}=0.39$, zoomed-in stimuli evoking a faster response than zoomed-out stimuli (423 ms versus $435 \mathrm{~ms})$; and, essentially for the research question, the Proximity $\mathrm{x}$ Threat interaction, $F(1,83)=$ $63, p<.0001, \eta_{\mathrm{p}}{ }^{2}=0.43$, due to the effect of Threat only being significant for the zoomed-in stimuli, $F(1,83)=100, p<.0001, \eta_{\mathrm{p}}^{2}=0.55(413 \mathrm{~ms}$ versus $433 \mathrm{~ms})$.

On accuracy, effects were found of Go, $F(1,83)=7.8, p=.0064, \eta_{\mathrm{p}}{ }^{2}=0.086$, Go responses being more accurate than Nogo responses (.92 versus .90); Proximity, $F(1,83)=18, p<.0001$, $\eta_{\mathrm{p}}{ }^{2}=0.17$, responses to zoomed-in stimuli being more accurate than responses to zoomed-out stimuli (.92 versus .91); Go x Threat, $F(1,83)=35, p<.0001, \eta_{\mathrm{p}}{ }^{2}=0.30$, due to the effect of Go being significant only for Threat stimuli, $F(1,83)=26, p<.0001, \eta_{\mathrm{p}}{ }^{2}=0.24$; Proximity $\mathrm{x}$ Threat, $F(1,83)=32, p<.0001, \eta_{\mathrm{p}}{ }^{2}=0.28$, the effect of Angry versus Neutral faces reversing for zoomed-out (lower accuracy for Angry faces, .89 versus .92) versus zoomed-in faces (higher accuracy for Angry faces, .93 versus .92); and Go x Proximity x Threat, $F(1,83)=7.5, p=$ $.0075, \eta_{\mathrm{p}}{ }^{2}=0.083$. For zoomed-out faces, there was a Go $\mathrm{x}$ Threat interaction, $F(1,83)=40, p<$ $.0001, \eta_{\mathrm{p}}{ }^{2}=0.32$, due to an effect of Threat for Nogo trials only, with more commission errors 
312 for Angry faces. For zoomed-in faces, there was also a Go x Threat interaction, , $F(1,83)=8.1$,

$313 p=.0056, \eta_{\mathrm{p}}{ }^{2}=0.089$, due to higher accuracy for Angry than Neutral faces for Go trials only.

314 3.3. Between-Task Comparisons

315 The above descriptive differences between task versions were tested using the mixed design

316 ANOVA. On RT, the following interactions were found, all due to the within-subject effect

317 being stronger in the Task-Relevant task version than in the Task-Irrelevant task version: Task

318 version $\mathrm{x}$ Threat, $F(1,171)=15, p=.00012, \eta_{\mathrm{p}}^{2}=0.083$; Task version $\mathrm{x}$ Proximity, $F(1,171)=$

$3199.9, p=.0020, \eta_{\mathrm{p}}^{2}=0.055$; Task-Version $\mathrm{x}$ Proximity $\mathrm{x}$ Threat, $F(1,171)=30, p<.0001, \eta_{\mathrm{p}}{ }^{2}=$ $320 \quad 0.15$.

321 On accuracy, the following interaction effects were found, all due to the within-subject effect

322 being significant only for the Task-Relevant task version: Task-Version x Go x Threat, $F(1,171)$

$323=11, p=.00092, \eta_{\mathrm{p}}^{2}=0.062$; Task-Version $\mathrm{x}$ Proximity $\mathrm{x}$ Threat, $F(1,171)=17, p=.00053$,

$324 \eta_{\mathrm{p}}^{2}=0.091$; Task-Version $\mathrm{x}$ Go x Proximity x Threat, $F(1,171)=6.2, p=.014, \eta_{\mathrm{p}}{ }^{2}=0.035$.

\section{4. Discussion}

326 The aims of the Study 2 were to test the effect of virtual stimulus proximity. The results also

327 allowed a conceptual replication of the task-relevance effect on impulsivity found in Study 1.

328 Threat-effects were again only found in the task-relevant version. Proximity was found to be

329 related to enhanced effects of threat on impulsivity, but only for the Task-Relevant task version

330 and most clearly for RT. This proximity effect for RT is in line with the defensive cascade

331 (Blanchard et al., 2001, 2005; Bradley, 2009; Heesink et al., 2017; Mobbs et al., 2007), in which

332 defensive responses depend on the distance to the threat. A threat appearing close by naturally

333 requires faster responses to escape, as an attack at shorter distance leaves less time to respond. It

334 would therefore be expected that proximity would enhance threat-induced impulsivity, as 
335 suggested by the RT results. Although an interaction was also found for accuracy, the pattern of

336 these results was more difficult to interpret. The expected increase in commission errors for

337 angry versus neutral faces was found for distant rather than nearby stimuli; while, more in line

338 with expectations, for nearby stimuli fewer false negatives were found for angry versus neutral

339 faces. One post-hoc interpretation of this phenomenon could be that the nearby presentation of

340 faces has an effect of enhancing attentional engagement and thereby improving accuracy, but

341 clearly this must be considered only speculative.

342 Study 3

$343 \quad 2$. Method

344 2.1.Participants

345 Healthy adult participants were recruited and received study credits for completing the study.

346 Participants gave informed consent. The study was approved by the ethics review board. 95

347 participants completed the experiment ( 79 female, 16 male; 21 years, $S D=2.7$ ) with accuracy

348 above .5 on all conditions ( $n=6$ participants were removed).

349

350 351 trials per block were 24.

\subsection{Emotional Go/Nogo Task (emoGNG)}

The same tasks as in Study 1 was used. The number of blocks per task was 5, and the number of

\section{2.3. Procedure}

353 Inclusion proceeded via an online participant-pool system. Participants could sign up for the

354 study based on a brief description, after which they could read the extensive information and 355 decide whether to continue. Participants performed both of the emoGNG versions, in random 356 order. Other questionnaires and tasks were performed in the same session that were related to 357 other studies. 
358 2.4. Preprocessing and Statistical Analyses

359 Preprocessing and analyses were the same as in Study 1, with the exception of task version now

360 being a within-subject variable.

$361 \quad 3$. Results

362 Descriptive statistics are presented in Table 3. 
364 Table 3. RT and accuracy on the emoGNG, within-subject design

365 3A. Reaction time on Go trials

\begin{tabular}{lll} 
Task version & Emotion & RT (SD) \\
\hline Task-irrelevant & Neutral & $450(29)$ \\
& Angry & $452(28)$ \\
Task-relevant & Neutral & $423(30)$ \\
& Angry & $417(31)$
\end{tabular}

366

367 3B. Accuracy

\begin{tabular}{llll} 
Task version & Emotion & Go/Nogo & Accuracy \\
\hline Task-irrelevant & Neutral & Nogo & .93 \\
& & Go & .95 \\
& Angry & Nogo & .91 \\
Task-relevant & Neutral & Go & .95 \\
& & Nogo & .90 \\
& Angry & Go & .92 \\
& & Nogo & .87 \\
& & Go & .93
\end{tabular}

368

369 Note. Mean and standard deviation of reaction time in ms and mean accuracy in proportion correct per

370 condition of the emoGNG over participants. Task version refers to task-relevance of the emotional

371 expression of the faces (Neutral or Angry).

372 


\subsection{Task-Irrelevant emoGNG}

There was no effect of Threat on RT and no interaction between Go and Threat on accuracy ( $p=$ .11). Go trials were more accurate than Nogo trials, $F(1,94)=30, p<.0001, \eta_{\mathrm{p}}{ }^{2}=0.24(.95$ versus .92). Angry trials were less accurate than Neutral trials, $F(1,94)=5.5, p=0.021, \eta_{\mathrm{p}}{ }^{2}=$ $0.056(.93$ versus .94$)$.

\subsection{Task-Relevant emoGNG}

On RT, there was an effect of Threat, $F(1,94)=9, p=.0035, \eta_{\mathrm{p}}{ }^{2}=0.087$, responding to Angry faces being faster than responding to Neutral faces (417 ms versus $423 \mathrm{~ms}$ ).

On accuracy, there was an interaction between Go and Threat, $F(1,94)=14, p=.0003, \eta_{\mathrm{p}}^{2}=$ 0.13. This was due to lower accuracy for Angry than Neutral faces on Nogo trials, $F(1,94)=10$, $p=.00017, \eta_{\mathrm{p}}{ }^{2}=0.099(.92$ versus .93 proportion correct), but higher accuracy on Go trials, $F(1$, $94)=4.6, p=.034, \eta_{\mathrm{p}}{ }^{2}=0.047$ (.93 versus .92 proportion correct). Further, Go trials were more accurate than Nogo trials, $F(1,94)=31, p<.0001, \eta_{\mathrm{p}}{ }^{2}=0.25(.93$ versus .89$)$.

\subsection{Between-Task Comparisons}

The above difference in effects between the tasks were formally tested using a repeated measures ANOVA. On RT, the interaction between Task version and Threat was significant, $F(1,94)=14$, $p=.00027, \eta_{\mathrm{p}}^{2}=0.13$. On accuracy, the interaction between Task version, Go, and Threat was significant, $F(1,94)=4.9, p=.029, \eta_{\mathrm{p}}{ }^{2}=0.05$.

\section{Discussion}

The results replicated the main pattern of effects from Study 1, but in a within-subject rather than between-subject design. Again, only in the task-relevant task version were threat stimuli associated with faster responses. Further, the Threat $x$ Go interaction was only found in the task- 
395 relevant version. The results of Study 3 this provide an important bridge to Study 4, in which 90-

39610 Go-Nogo proportions were used in a within-subject design.

397 Study 4

398 2. Method

$3992.1 . P a r t i c i p a n t s$

400 Healthy adult participants were recruited and received study credits for completing the study.

401 Participants gave informed consent. The study was approved by the ethics review board. 46

402 participants completed the experiment (40 female, 6 male, 21 years, $S D=6.2$ ), with a minimum

403 accuracy of .1 in all conditions. The minimum accuracy criterion used in previous studies (with

404 equal go and nogo frequencies) was found to be too strict in this task variant, leading to rejection

405 of the majority of participants. This was due to a large increase in the rate of commission errors.

406 The more lenient criterion was used in order to attempt to restrict removal to participants who

407 were most likely failing to try to inhibit responses at all $(n=6)$.

408 2.2. Emotional Go/Nogo Task (emoGNG)

409 The same tasks as in Study 3 were used, but with a 90\% go, 10\% nogo rate. For each task

410 version, there was a practice task with 2 blocks of 24 trials. The full assessment versions of the

411 tasks had 10 blocks of 24 trials.

412 2.3. Procedure

413 Inclusion proceeded via an online participant-pool system. Participants could sign up for the

414 study based on a brief description, after which they could read the extensive information and

415 decide whether to continue. Participants performed short practice versions of both emoGNG

416 versions, and then assessment versions of both emoGNG versions, with the order of task-

417 relevance randomized per participant. 
418 2.4. Preprocessing and Statistical Analyses

419 The preprocessing and analyses were identical to Study 3. Only the assessment versions were

420 used for analysis.

$421 \quad 3$. Results

422 Descriptive statistics are presented in Table 4. 
424 Table 4. RT and accuracy on the emoGNG, 90-10 go-nogo rates version

425 4A. Reaction time on Go trials

\begin{tabular}{lll} 
Task version & Emotion & RT (SD) \\
\hline Task-irrelevant & Neutral & $416(39)$ \\
& Angry & $417(38)$ \\
Task-relevant & Neutral & $361(45)$ \\
& Angry & $362(43)$
\end{tabular}

427 4B. Accuracy

\begin{tabular}{llll} 
Task version & Emotion & Go/Nogo & Accuracy \\
\hline Task-irrelevant & Neutral & Nogo & .56 \\
& & Go & .97 \\
& Angry & Nogo & .55 \\
Task-relevant & Neutral & Go & .97 \\
& & Nogo & .52 \\
& Angry & Go & .97 \\
& & Nogo & .53 \\
& & Go & .96
\end{tabular}

428

429 Note. Mean and standard deviation of reaction time in ms and mean accuracy in proportion correct per

430 condition of the emoGNG over participants. Task version refers to task-relevance of the emotional

431 expression of the faces (Neutral or Angry).

432 


\section{3.1. Task-Irrelevant emoGNG}

434 There was no effect of Threat on RT ( $p=.093$, direction of effect in reversed direction) and no

435 interaction between Go and Threat on accuracy $(p=.86)$. Go trials were more accurate than

436 Nogo trials, $F(1,45)=520, p<0.0001, \eta_{\mathrm{p}}{ }^{2}=0.92(.97$ versus .56$)$.

\section{3.2. Task-Relevant emoGNG}

438 There was no effect of Threat on RT $(p=.76)$ and no interaction between Go and Threat on

439 accuracy $(p=.12)$. Go trials were more accurate than Nogo trials, $F(1,45)=400, p<0.0001, \eta_{\mathrm{p}}^{2}$

$440=0.90(.97$ versus .53$)$.

441 3.3. Between-Task Comparisons

442 There were no interactions involving task version.

443 4. Discussion

444 With 90-10 rates of go and nogo trials, there was no sign of the threat-related effects found in

445 previous studies. This was the case for both the task-relevant and task-irrelevant version. We

446 reiterate one of the reasons for using equal versus unequal rates: the block-context strongly

447 differs when Threat is mapped to go versus nogo responses (e.g., the frequency of Angry versus

448 Neutral faces changes along with the current block's task instructions), which may well interact

449 with effects of trial type. While there are clearly many possible variations involving go - nogo

450 rates, the current study's rationale and results would appear to suggest that using 50-50 rates

451 should be considered a potentially interesting and valid design choice. The consistent threat-

452 related results found for the task-relevant version with 50-50 rates were lost with the 90-10 rates,

453 and there is no indication that this change revealed threat-related effects that were absent in the

454 previous task-irrelevant versions. 


\section{General Discussion}

The current studies aimed to determine whether threat induces impulsivity as reflected in both speeding and commission errors on a Go-Nogo task. A number of task design choices were explored. As discussed in the introduction, there were various reasons to choose equal rates for go and nogo frequencies, and the null results of Study 4, which used 90-10 rates in contrast with the other three studies, suggest that the 50-50 design is more sensitive to threat effects. In the first three studies, but only in the task-relevant versions, the presence of angry faces caused faster responses and more commission errors. This is in line with a reduction in response threshold induced by threatening stimuli, as would be expected from their evolutionary significance. No significant effects involving threat-induced impulsivity were found in the taskirrelevant versions. It may be the case that the automatic bias due to threatening stimuli only induces impulsivity when the inducing stimuli are task-relevant, as has been found in previous work, with various broadly related conceptualizations of task-relevance (Lichtenstein-Vidne et al., 2012; Spruyt et al., 2009, 2018). Note that this does not entail a "non-automatic" effect participants were not instructed to respond faster to Threat stimuli, but this occurred automatically when they had to process emotional information to perform the task. It may also be the case that when distractors were task-irrelevant, the effect of the facial expression was muted via selective attention. The ability to suppress, or treat as irrelevant, potentially distracting emotional information has been speculated to play a conceptually similar role in various effects related to attentional biases (Gladwin, 2017; Gladwin, Ter Mors-Schulte, Ridderinkhof, \& Wiers, 2013). In this case, the ability to tune out task-irrelevant, potentially distracting information could reduce threat-evoked effects on task-irrelevant Go-Nogo tasks. 
477 The impact of having the threatening stimuli appear to have closer proximity was as predicted

478 for reaction times, although, again, effects required task-relevant stimuli. Although effects on

479 accuracy were more difficult to interpret, relative proximity increased threat-induced speeding.

480 This was expected given the view of a natural, evolutionarily preserved tendency to respond

481 quickly, and hence with less extensive evaluation of response selection, to nearby threatening

482 stimuli (Blanchard et al., 2001, 2005; Bradley, 2009). Proximal threat evokes

483 psychophysiological activity related to acute emotional-physiological responses to threat (Löw,

484 Lang, Smith, \& Bradley, 2008; Mobbs et al., 2007). In line with this, neuroimaging results from

485 the Fear and Escape Task (Montoya, Terburg, Bos, \& van Honk, 2012) in a population of

486 veterans indicate that abnormal reactions to proximity may be involved in anger and aggression

487 problems (Heesink et al., 2017). A “looming” stimulus (Vagnoni, Lourenco, \& Longo, 2012)

488 was found to evoke abnormally strong activation in attention-related brain regions in participants

489 with anger and aggression problems. It would appear that anger disorders are a particularly

490 worthwhile clinical focus of further study of proximity-enhanced, threat-induced speeding.

491 The current study had a number of limitations. First, a sample of students was used for pragmatic

492 reasons, rather than, e.g., potentially interesting clinical or forensic groups. It is possible that

493 different effects would be found in groups with more dysfunctional responses to threat. Second,

494 the study was online, which reduces the ability to control the testing environment, but has clear

495 practical advantages in terms of the efficiency of acquiring data. In future studies, in particular

496 using clinical populations, a different trade-off of concerns could indicate the use of laboratory

497 settings. Third, although the results of Study 4 appear to point in a clear direction supporting the

498 use of equal probabilities in this context, it is not certain to which extent the results will or will

499 not generalize to Go/Nogo tasks with other specific proportions of nogo trials. Fourth, the 
500 numbers of blocks and trials were slightly different in different studies. There was no principled

501 reason for the precise trial numbers, but this minor difference would not seem to substantially

502 affect any conclusions drawn from the studies. Fifth, the study was focused on a specific

503 stimulus type, namely faces with angry versus neutral expressions. While this was a conscious

504 feature of the study and specifically extends the literature on emotional Go/Nogo tasks to these

505 stimuli, the current results cannot say whether the differences between the Emotion-Relevant and

506 Emotion-Irrelevant task versions will generalize to different stimuli. We also cannot specify the

507 precise feature of the threatening stimuli that induced impulsivity, e.g., whether the angry faces

508 were more arousing or more negative (note that threat itself as a concept is related to both

509 arousal and negative valence). There is clearly scope for many lines of future research, exploring

510 many more variations of task design and parameters; however, the current results provide a proof

511 of principle that at least using the current stimuli and task parameters, task-relevance affects

512 impulsivity evoked by stimuli involving threat.

513 In conclusion, angry versus neutral faces are able to induce impulsive responding, but significant

514 effects were only found when these emotional stimuli were task-relevant and when go and nogo

515 trials were equally frequent. With this task version, partial support was found in RT effects for

516 the hypothesis that threat-induced impulsivity would be enhanced by increasing the perceived

517 proximity of the threatening stimulus. Future research in which effects of impulsivity on RT are

518 of interest could consider using this task design.

$519 \quad$ Funding

520 This research did not receive any specific grant from funding agencies in the public, commercial, 521 or not-for-profit sectors. 


\section{Declaration of conflicting interests}

523 The Authors declare that there is no conflict of interest.

524

525

526

527

528

529

530

531

532

533

534

\section{References}

Bargh, J. A. (1994). The four horsemen of automaticity: Awareness, intention, efficiency and control in social cognition. In R. Wyer \& T. Srull (Eds.), Handbook of Social Cognition (2nd ed., pp. 1-40). Hillsdale, NJ: Erlbaum.

Bargh, J. A., \& Ferguson, M. J. (2000). Beyond behaviorism: On the automaticity of higher mental processes. Psychological Bulletin, 126(6), 925-945. https://doi.org/10.1037//00332909.126.6.925

Bari, A., \& Robbins, T. W. (2013). Inhibition and impulsivity: Behavioral and neural basis of response control. Progress in Neurobiology, 108, 44-79. https://doi.org/10.1016/j.pneurobio.2013.06.005

Bishop, S. J. (2008). Neural mechanisms underlying selective attention to threat. Annals of the New York Academy of Sciences, 1129, 141-52. https://doi.org/10.1196/annals.1417.016

Blanchard, D. C., Blanchard, R. J., \& Griebel, G. (2005). Defensive Responses to Predator Threat in the Rat and Mouse. Current Protocols in Neuroscience, 30(1), 8.19.1-8.19.20. https://doi.org/10.1002/0471142301.ns0819s30

Blanchard, D. C., Griebel, G., Pobbe, R., \& Blanchard, R. J. (2011). Risk assessment as an evolved threat detection and analysis process. Neuroscience and Biobehavioral Reviews, 35(4), 991-8. https://doi.org/10.1016/j.neubiorev.2010.10.016

Blanchard, D. C., Hynd, A. L., Minke, K. A., Minemoto, T., Blanchard, R. J., Caroline 
Blanchard, D., ... Blanchard, R. J. (2001). Human defensive behaviors to threat scenarios show parallels to fear- and anxiety-related defense patterns of non-human mammals. Neuroscience and Biobehavioral Reviews, 25(7-8), 761-70. https://doi.org/10.1016/S01497634(01)00056-2

Bracha, H. S. (2004). Freeze, flight, fight, fright, faint: adaptationist perspectives on the acute stress response spectrum. CNS Spectrums, 9(9), 679-85. Retrieved from http://www.ncbi.nlm.nih.gov/pubmed/15337864

Bradley, M. M. (2009). Natural selective attention: orienting and emotion. Psychophysiology, 46(1), 1-11. https://doi.org/10.1111/j.1469-8986.2008.00702.x

Brown, M. R. G., Benoit, J. R. A., Juhás, M., Lebel, R. M., MacKay, M., Dametto, E., ... Greenshaw, A. J. (2015). Neural correlates of high-risk behavior tendencies and impulsivity in an emotional Go/NoGo fMRI task. Frontiers in Systems Neuroscience, 9, 24. https://doi.org/10.3389/fnsys.2015.00024

Chetverikov, A., \& Upravitelev, P. (2016). Online versus offline: The Web as a medium for response time data collection. Behavior Research Methods, 48(3), 1086-99. https://doi.org/10.3758/s13428-015-0632-x

Coombes, S. A., Tandonnet, C., Fujiyama, H., Janelle, C. M., Cauraugh, J. H., \& Summers, J. J. (2009). Emotion and motor preparation: A transcranial magnetic stimulation study of corticospinal motor tract excitability. Cognitive, Affective \& Behavioral Neuroscience, 9(4), 380-8. https://doi.org/10.3758/CABN.9.4.380

Cunningham, W. A., Zelazo, P. D., Packer, D. J., \& Van Bavel, J. J. (2007). The Iterative 
Reprocessing Model: A Multilevel Framework for Attitudes and Evaluation. Social Cognition, 25(5), 736-760. https://doi.org/10.1521/soco.2007.25.5.736

De Houwer, J., Teige-Mocigemba, S., Spruyt, A., \& Moors, A. (2009). Implicit measures: A normative analysis and review. Psychological Bulletin, 135(3), 347-68. https://doi.org/10.1037/a0014211

De Houwer, J., \& Tibboel, H. (2010). Stop what you are not doing! Emotional pictures interfere with the task not to respond. Psychonomic Bulletin \& Review, 17(5), 699-703. https://doi.org/10.3758/PBR.17.5.699

de Vignemont, F., \& Iannetti, G. D. (2015). How many peripersonal spaces? Neuropsychologia, 70, 327-334. https://doi.org/10.1016/J.NEUROPSYCHOLOGIA.2014.11.018

Fanselow, M. S. (1986). Associative vs topographical accounts of the immediate shock-freezing deficit in rats: Implications for the response selection rules governing species-specific defensive reactions. Learning and Motivation, 17(1), 16-39. Retrieved from http://www.sciencedirect.com/science/article/pii/0023969086900184

Gladwin, T. E. (2017). Negative effects of an alternating-bias training aimed at attentional flexibility: A single session study. Health Psychology and Behavioral Medicine, 5(1), 4156. https://doi.org/10.1080/21642850.2016.1266634

Gladwin, T. E., Hashemi, M. M., van Ast, V. A., \& Roelofs, K. (2016). Ready and waiting: Freezing as active action preparation under threat. Neuroscience Letters, 619, 182-188. https://doi.org/10.1016/j.neulet.2016.03.027

Gladwin, T. E., Ter Mors-Schulte, M. H. J., Ridderinkhof, K. R., \& Wiers, R. W. (2013). Medial 
parietal cortex activation related to attention control involving alcohol cues. Frontiers in Psychiatry, 4, 174. https://doi.org/10.3389/fpsyt.2013.00174

Graziano, M. S. A., \& Cooke, D. F. (2006). Parieto-frontal interactions, personal space, and defensive behavior. Neuropsychologia, 44(6), 845-859. https://doi.org/10.1016/J.NEUROPSYCHOLOGIA.2005.09.009

Hartikainen, K. M., Siiskonen, A. R., \& Ogawa, K. H. (2012). Threat interferes with response inhibition. Neuroreport, 23(7), 447-50. https://doi.org/10.1097/WNR.0b013e3283531e74

Hayduk, L. A. (1983). Personal space: Where we now stand. Psychological Bulletin, 94(2), 293335. https://doi.org/10.1037/0033-2909.94.2.293

Heesink, L., Gladwin, T. E., Terburg, D., van Honk, J., Kleber, R., \& Geuze, E. (2017). Proximity alert! Distance related cuneus activation in military veterans with anger and aggression problems. Psychiatry Research: Neuroimaging, 266, 114-122. https://doi.org/10.1016/j.pscychresns.2017.06.012

Kozlowska, K., Walker, P., McLean, L., \& Carrive, P. (2015). Fear and the Defense Cascade: Clinical Implications and Management. Harvard Review of Psychiatry, 23(4), 263-87. https://doi.org/10.1097/HRP.0000000000000065

Krypotos, A.-M., Beckers, T., Kindt, M., \& Wagenmakers, E.-J. (2015). A Bayesian hierarchical diffusion model decomposition of performance in Approach-Avoidance Tasks. Cognition and Emotion, 29(8), 1424-1444. https://doi.org/10.1080/02699931.2014.985635

Lichtenstein-Vidne, L., Henik, A., \& Safadi, Z. (2012). Task relevance modulates processing of distracting emotional stimuli. Cognition \& Emotion, 26(1), 42-52. 
Löw, A., Lang, P. J., Smith, J. C., \& Bradley, M. M. (2008). Both predator and prey: emotional arousal in threat and reward. Psychological Science, 19(9), 865-73. https://doi.org/10.1111/j.1467-9280.2008.02170.x

McGurk, B. J., Davis, J. D., \& Grehan, J. (1981). Assaultive behavior personality and personal space. Aggressive Behavior, 7(4), 317-324. https://doi.org/10.1002/10982337(1981)7:4<317::AID-AB2480070402>3.0.CO;2-G

Megías, A., Gutiérrez-Cobo, M. J., Gómez-Leal, R., Cabello, R., \& Fernández-Berrocal, P. (2017). Performance on emotional tasks engaging cognitive control depends on emotional intelligence abilities: an ERP study. Scientific Reports, 7(1), 16446. https://doi.org/10.1038/s41598-017-16657-y

Mobbs, D., Petrovic, P., Marchant, J. L., Hassabis, D., Weiskopf, N., Seymour, B., ... Frith, C. D. (2007). When fear is near: Threat imminence elicits prefrontal-periaqueductal gray shifts in humans. Science (New York, N.Y.), 317(5841), 1079-83. https://doi.org/10.1126/science. 1144298

Montoya, E. R., Terburg, D., Bos, P. A., \& van Honk, J. (2012). Testosterone, cortisol, and serotonin as key regulators of social aggression: A review and theoretical perspective. Motivation and Emotion, 36(1), 65-73. https://doi.org/10.1007/s11031-011-9264-3

Nieuwenhuys, A., \& Oudejans, R. R. D. (2012). Anxiety and perceptual-motor performance: Toward an integrated model of concepts, mechanisms, and processes. Psychological Research. https://doi.org/10.1007/s00426-011-0384-x 
627 Nieuwenhuys, A., Savelsbergh, G. J. P., \& Oudejans, R. R. D. (2012). Shoot or don’t shoot?

628

629

630

631

632

633

634

635

636

637

638

639

640

641

642

643

644

645

646

647 Why police officers are more inclined to shoot when they are anxious. Emotion, 12(4), 82733. https://doi.org/10.1037/a0025699

Oei, N. Y. L., Veer, I. M., Wolf, O. T., Spinhoven, P., Rombouts, S. A. R. B., \& Elzinga, B. M. (2012). Stress shifts brain activation towards ventral 'affective' areas during emotional distraction. Social Cognitive and Affective Neuroscience, 7(4), 403-412. https://doi.org/10.1093/scan/nsr024

Pawliczek, C. M., Derntl, B., Kellermann, T., Kohn, N., Gur, R. C., \& Habel, U. (2013). Inhibitory control and trait aggression: Neural and behavioral insights using the emotional stop signal task. NeuroImage, 79, 264-274. Retrieved from http://www.sciencedirect.com/science/article/pii/S1053811913004631

Pessoa, L., McKenna, M., Gutierrez, E., \& Ungerleider, L. G. (2002). Neural processing of emotional faces requires attention. Proceedings of the National Academy of Sciences of the United States of America, 99(17), 11458-63. https://doi.org/10.1073/pnas.172403899

Roelofs, K. (2017). Freeze for action: Neurobiological mechanisms in animal and human freezing. Philosophical Transactions of the Royal Society B: Biological Sciences, 372(1718), 20160206. https://doi.org/10.1098/rstb.2016.0206

Sagaspe, P., Schwartz, S., \& Vuilleumier, P. (2011). Fear and stop: a role for the amygdala in motor inhibition by emotional signals. NeuroImage, 55(4), 1825-35. https://doi.org/10.1016/j.neuroimage.2011.01.027

Sagliano, L., Cappuccio, A., Trojano, L., \& Conson, M. (2014). Approaching threats elicit a 
freeze-like response in humans. Neuroscience Letters, 561, 35-40. https://doi.org/10.1016/j.neulet.2013.12.038

Sambo, C. F., \& Iannetti, G. D. (2013). Better safe than sorry? The safety margin surrounding the body is increased by anxiety. The Journal of Neuroscience : The Official Journal of the Society for Neuroscience, 33(35), 14225-30. https://doi.org/10.1523/JNEUROSCI.070613.2013

Schutter, D. J. L. G., Hofman, D., \& Van Honk, J. (2008). Fearful faces selectively increase corticospinal motor tract excitability: A transcranial magnetic stimulation study. Psychophysiology, 45(3), 345-348. https://doi.org/10.1111/j.1469-8986.2007.00635.x

Spruyt, A., De Houwer, J., \& Hermans, D. (2009). Modulation of automatic semantic priming by feature-specific attention allocation. Journal of Memory and Language, 61(1), 37-54. https://doi.org/10.1016/J.JML.2009.03.004

Spruyt, A., Tibboel, H., De Schryver, M., \& De Houwer, J. (2018). Automatic stimulus evaluation depends on goal relevance. Emotion, 18(3), 332-341. https://doi.org/10.1037/emo0000361

Thoma, P., Soria Bauser, D., \& Suchan, B. (2013). BESST (Bochum Emotional Stimulus Set)— A pilot validation study of a stimulus set containing emotional bodies and faces from frontal and averted views. Psychiatry Research, 209(1), 98-109. https://doi.org/10.1016/j.psychres.2012.11.012

Vagnoni, E., Lewis, J., Tajadura-Jiménez, A., \& Cardini, F. (2018). Listening to a conversation with aggressive content expands the interpersonal space. PLOS ONE, 13(3), e0192753. 

https://doi.org/10.1371/journal.pone.0192753

670 Vagnoni, E., Lourenco, S. F., \& Longo, M. R. (2012). Threat modulates perception of looming 671 visual stimuli. Current Biology : $C B, 22(19), \mathrm{R} 826-7$.

672 https://doi.org/10.1016/j.cub.2012.07.053

673 van Ballegooijen, W., Riper, H., Cuijpers, P., van Oppen, P., \& Smit, J. H. (2016). Validation of 674 online psychometric instruments for common mental health disorders: a systematic review. 675 BMC Psychiatry, 16(1), 45. https://doi.org/10.1186/s12888-016-0735-7

676 van Peer, J. M., Gladwin, T. E., \& Nieuwenhuys, A. (2018). Effects of Threat and Sleep

677 Deprivation on Action Tendencies and Response Inhibition. Emotion, In press. $678 \quad$ https://doi.org/10.1037/emo0000533

679 Verbruggen, F., \& De Houwer, J. (2007). Do emotional stimuli interfere with response 680 inhibition? Evidence from the stop signal paradigm. Cognition \& Emotion, 21(2), 391-403. 681 https://doi.org/10.1080/02699930600625081

682 Verbruggen, F., \& Logan, G. D. (2008). Response inhibition in the stop-signal paradigm. Trends 683 in Cognitive Sciences, 12(11), 418-24. https://doi.org/10.1016/j.tics.2008.07.005 
686 Figure 1. Illustration of stimuli during the Emotional Go-Nogo training task

A

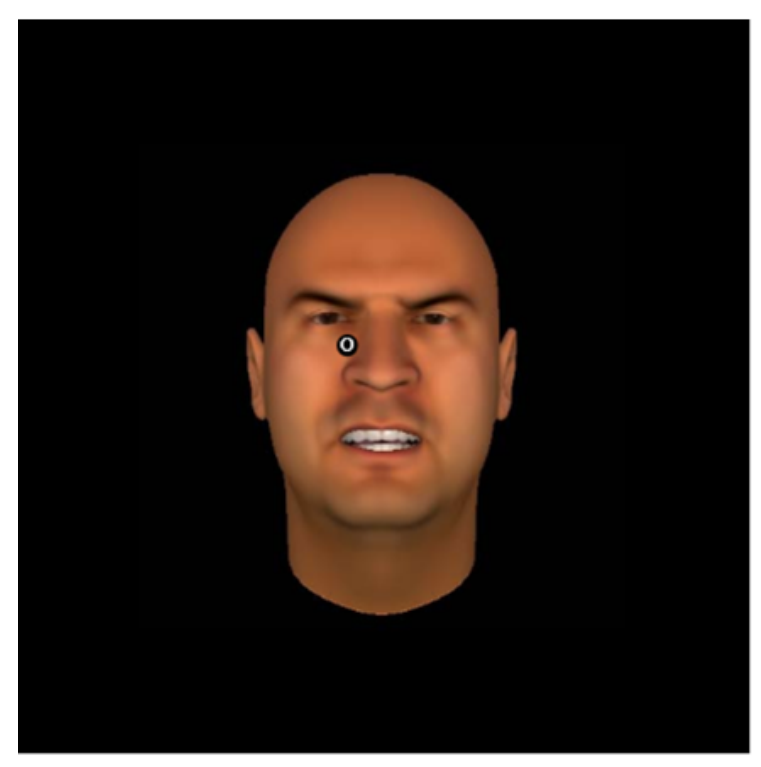

B

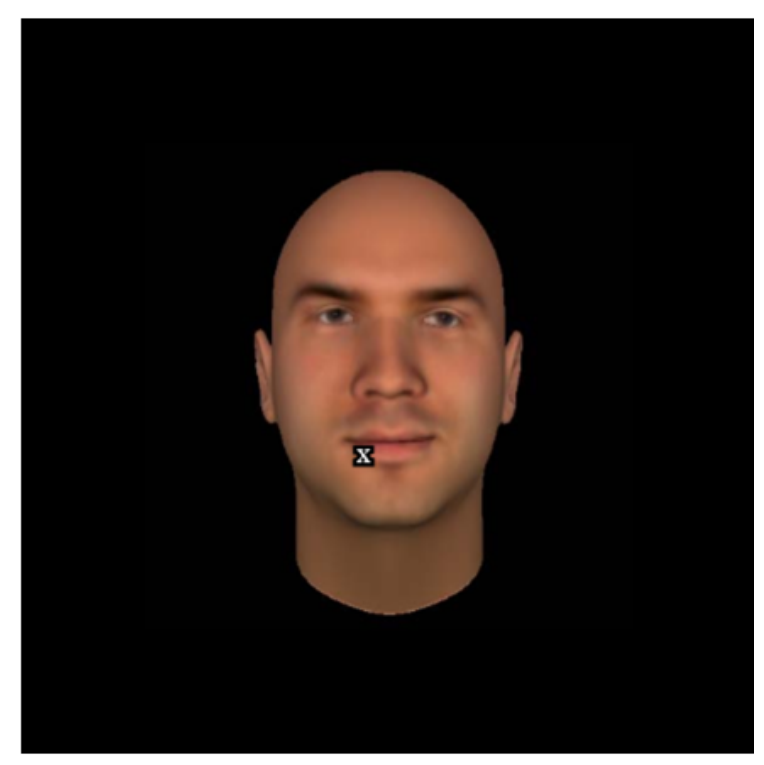

688 Note. Stimuli were an Angry or Neutral face with an X or an O superimposed at a random

689 location. Figures A and B show examples of an Angry face with an $\mathrm{O}$ and a Neutral face 690 with an $\mathrm{X}$, respectively. 